\title{
Qualität und Ethik - Beiträge zur guten Gesundheitsversorgung
}

\author{
Gerald Neitzke $\cdot$ Daniel Strech
}

Online publiziert: 11. Juli 2017

(C) Springer-Verlag GmbH Deutschland 2017

„Hohe Qualität“ ist ein Gütezeichen von Gesundheits- und anderen sozialen Leistungen. Das betrifft die ärztliche und pflegerische Versorgung von Menschen in Krankenhäusern, Pflegeeinrichtungen oder Hospizen genauso wie in der ambulanten Versorgung. Aber auch die gesundheitsbezogene Forschung kann nur durch hohe Qualitätsstandards als moralisch gerechtfertigt gelten. Es gibt mindestens zwei unterschiedliche Ursachen für dieses Qualitätsbewusstsein und diese Qualitätserwartung: Die starke intrinsische (ethische) Motivation der Anbieter von Gesundheitsleistungen, dies bestmöglich zu tun. Und natürlich die Erwartungen von den Hilfebedürftigen und ihren Kostenträgern, dass die Leistungen einerseits auf höchstem fachlichen Niveau und andererseits kosteneffizient erbracht werden. Beide Ursachen spielen bei der Qualitätsdiskussion eine erhebliche Rolle.

Der Begriff „Qualität“ lässt zunächst zwei unterschiedliche Lesarten zu. Zum einen werden als Qualitäten die Merkmale und Eigenschaften von Prozessen und Strukturen bezeichnet. Dieser Qualitätsbegriff ist also zunächst rein deskriptiv und wertneutral. Zum anderen beschreibt „Qualität“ aber auch die Güte von Prozessen und Strukturen und ist in dieser Bedeutung eindeutig normativ, da auf einen Begriff des Guten zurückgegriffen wird. Schon an dieser Stelle wird deutlich, dass der Ethik eine zentrale Rolle bei Qualitätsdiskussionen zukommen muss, da diese Diskussionen eine Verständigung über das Gute selbst, bzw. geeignete und interindividuell vermittelbare Gütekriterien voraussetzt.

Bezogen auf den Diskurs über Qualität im Gesundheitswesen ist also ein Beitrag der Medizin- und Pflegeethik unverzichtbar. Dies steht jedoch der Erfahrung diametral entgegen, dass viele Maßnahmen etwa des Qualitätsmanagements i. d. R.

Dr. med. G. Neitzke $(\bowtie) \cdot$ Prof. Dr. med. Dr. phil. D. Strech

Institut für Geschichte, Ethik und Philosophie der Medizin, Medizinische Hochschule Hannover,

Carl-Neuberg-Str. 1, 30625 Hannover, Deutschland

E-Mail: neitzke.gerald@mh-hannover.de 
nicht unter Hinzuziehung von Ethikern erarbeitet werden. Qualitätsmanagement ist im Gesundheitswesen mittlerweile weit verbreitet und wird auf der gesetzlichen Grundlage des $\S 135$ a Sozialgesetzbuch (SGB) V durchgeführt. Dabei sind weite Teile des Qualitätsmanagements auf eine Effizienzsteigerung im Rahmen der Leistungserbringung ausgerichtet. Das bedeutet, dass die Leistungen bei gleicher Qualität möglichst kostengünstig erwirtschaftet werden sollen. Selbstverständlich ist auch dies ein ethisches Gebot, da die Alternative ja eine Verschwendung von Ressourcen bedeuten würde; Ressourcen, die an anderer Stelle im Gesundheitswesen benötigt werden. Gleichzeitig reduziert dieses Verständnis die Qualitätsdiskussion auf das ökonomische Kriterium der Effizienz und verdeckt damit ihre weiteren ethischen Gehalte.

Deshalb sollten die ethischen Aspekte des Qualitätsbegriffs im Gesundheitswesen ausdrücklich formuliert und als Vorgaben für das Qualitätsmanagement festgelegt werden. Diesem Diskurs widmet sich die Jahrestagung der AEM 2017. Welches sind die ethischen Grundwerte, ohne die Qualität in der Gesundheitsversorgung nicht vorstellbar bzw. wünschenswert ist? Wieviel kann und muss uns die Realisierung dieser ethischen Aspekte wert sein? Welche Teile der ärztlichen, pflegerischen und therapeutischen Bemühungen lassen sich nicht in einer Effizienzkalkulation abbilden und wiederfinden, sind aber gleichwohl prägender Teil dieser Arbeit? Und als ,prägend“ verstehen wir an dieser Stelle diejenigen Aspekte und Qualitäten von sozialen Dienstleistungen, die entweder von den Hilfebedürftigen als ethisches Minimum im Umgang, in der Kommunikation, in der Zuwendung verstanden werden, oder die als professioneller Mindeststandard das eigene professionelle Bedürfnis ausdrücken, nicht weniger fürsorglich und verantwortungsvoll arbeiten $\mathrm{zu}$ wollen.

Sollte die erste Bedingung nicht erfüllt werden, schwindet das Vertrauen ins Gesundheitswesen und dessen gesellschaftliches Ansehen, wenn die zweite Bedingung nicht erfüllt ist, werden sich die gewissenhaften Anbieter von Gesundheitsleistungen frustriert vom Gesundheitswesen abwenden. Die ökonomisch orientierte Qualitätssicherung im Gesundheitswesen ist einerseits notwendig, darf andererseits nicht dazu führen, dass Ärzte und Pflegende dauerhaft gezwungen werden, unterhalb ihrer eigenen moralischen Ansprüche arbeiten zu müssen. Dies erodiert sowohl die professionelle Moral als auch langfristig die Qualität in der Medizin.

Die Jahrestagung in Hannover bietet deshalb ein Forum, dass alle Beteiligten und Betroffenen ihre Vorstellungen von Qualität darlegen und insbesondere die ethischen Beiträge zur Qualitätsdiskussion konkretisieren können. Was macht Pflege und Behandlung ,gut“, ab wann ist sie ,gut genug“? Welche Vorstellungen von einem guten Arzt, einer guten Ärztin, einer guten Kranken- oder Altenpflegerin haben wir? Wie können diese ethischen Qualitäten bereits in Studium und Berufsausbildung gefördert werden? Wie kann die Entscheidungsfindung in den Institutionen der Qualitätssicherung, also etwa dem Institut für Qualität und Wirtschaftlichkeit im Gesundheitswesen (IQWiG), dem Institut für Qualitätssicherung und Transparenz im Gesundheitswesen (IQTIG) oder dem Gemeinsamen Bundesausschuss (G-BA), durch ethische Expertise unterstützt und verbessert werden?

Dieselben Fragen lassen sich auch an die gesundheitsbezogene Forschung richten. Was macht wissenschaftliche Praxis ,gut“? Was erwarten wir von einer „guten“ Qualitätssicherung in der Forschung z. B. durch Ethikkommissionen? Was müssen 
die Forschungseinrichtungen tun, damit die Forschenden in der Lage sind, allgemein anerkannte ethische Standards in der Forschung in ihrer Forschungstätigkeit umzusetzen? Dies umfasst sowohl die Fortbildung als auch Arbeitsbedingungen, in denen ohne Angst vor Nachteilen ethische Standards eingehalten werden können.

Letztlich muss aber auch die Ethik selbst in ihren praktischen Gebieten eine Qualitätsdiskussion führen. Welche Kompetenzen und welchen Informationsstand müssen Ethikerinnen und Ethiker mitbringen, um die „Qualitätsakteure“ in Versorgung und Forschung zielführend zu unterstützen? Was ist gute Forschung in der Medizin- und Pflegeethik? Die professionelle Medizinethik hat insbesondere durch die Klinische Ethikberatung erheblich an Dynamik gewonnen. Weiterhin sind Ethikerinnen und Ethiker Teil einer institutionalisierten Ethik wie im Deutschen Ethikrat, in der Zentralen Ethikkommission bei der Bundesärztekammer oder in den Arbeitsgruppen der AEM. Welche (ethischen) Qualitätskriterien liegen diesen Initiativen und deren Produkten (wie z. B. Leitlinien, Positionierungen, Empfehlungen) zugrunde? Und auf welche Weise kann Qualitätssicherung auch in den Bereich der Ethikberatung und anderer Aufgaben von Ethikkomitees, -kommissionen, und -AGs Eingang finden?

„Es gibt nichts Gutes, außer: Man tut es“ hat Erich Kästner 1950 in seiner Sammlung „Kurz und bündig“ geschrieben. Darin steckt die Aufforderung, aber auch die tiefe Überzeugung, dass wir um dieses Gute immer neu ringen müssen. Die Definition des ethischen Anteils von Qualität in der Gesundheitsversorgung, aber auch die Ermöglichung dieser ethischen Standards in der Ausübung der Gesundheitsberufe ist zentrale Aufgabe der Ethik in der Medizin. Wir freuen uns auf Ihre Diskussionsbeiträge vom 28.-30. September 2017. 